\title{
Theoretical Analysis of Experimental Thermoelectric Characteristics of Silicon-Based Whiskers
}

\author{
Lviv Polytechnic National University, Lviv, Ukraine, druzh@polynet.lviv.ua
}

It is shown that when a conductive crystal with electric field strength $\stackrel{\mathrm{I}}{\mathrm{E}}$ and a temperature gradient $\nabla \underset{r}{\mathrm{r}} T$ is placed in a magnetic field with an induction vector $\stackrel{\prime}{B}$, processes of charge and heat carriers transport occur, and they can be described by known generalized electrical conduction and heat conduction equations. The tensors and scalar coefficients that make up these equations are the kinetic properties of crystals. They describe the nature of actual properties of crystals and have a wide pragmatic application in modern solid-state electronics. The process of spatial quantization of the spectrum and its influence on the kinetic properties of crystals is also analyzed.

Key words: Gibbs potential, chemical potential, dispersion law, whisker, thermoelectrics.

Article acted received 13.12.2019; accepted for publication15.12.2019.

\section{Introduction}

The well-known phenomenological theory of kinetic properties of the solid state gives an understanding of how to determine the main kinetic properties of semiconductor crystals, e.g. the energy spectrum $\varepsilon(\mathrm{p})$, the scattering function $u(\varepsilon)$, and the reduced chemical potential $\mu$ [1-4]. These properties allow predicting the characteristics of crystals and their potential application $[5,6]$. The kinetic properties of the semiconductor crystals can also be considered in the framework of the statistical theory of the solid state. In this paper, a theoretical analysis of experimental thermoelectric characteristics of silicon-based whiskers is done with the use of previously published kinetic theory model [7-9]. The data of thermoelectric characteristics of Si whiskers are taken from [10-12].

\section{Elements of the General Theory of Kinetic Properties of Crystals}

The kinetic properties of conductive crystals are determined by the concentration of free charge carriers in the crystals and the nature of their motion in the interstices of the crystal lattice.
In a state of thermodynamic equilibrium, free charge carriers move chaotically, their average energy is stored, and entropy of the entire charge carrier set is highly important. It is a thermodynamically equilibrium charge carrier gas.

The presence in the crystal of drift perturbations, namely, an electric field with intensity $\stackrel{E}{E}$, a temperature gradient $\nabla_{r} T$, (these perturbations can exist in the crystal simultaneously) causes the charge carrier gas to escape from the state of thermodynamic equilibrium and transform it into a nonequilibrium ensemble of particles. In this case, a drift force $\dot{\mathrm{F}}_{д}$ acts on each particle with ze charge [7-9]:

$$
\stackrel{r}{F_{d}}=z e E_{d} ; \quad \stackrel{r}{E}_{d}=\stackrel{r}{E}-\left(\frac{k}{z e}\right)\left(\frac{\varepsilon-\mu}{k T}\right) \nabla r r
$$

where $e$ - electron charge, $\mathrm{z}= \pm 1-$ charge sign, $\varepsilon-$ average charge energy, $k$ - Boltzmann constant, T crystal temperature.

Due to the action of the drift force $\dot{F}_{d}$, all charge carriers begin to move at a drift velocity $\dot{v}_{d}$ that depends on both, $\dot{F}_{d}$ and the crystal properties. The presence of a drift velocity $\stackrel{1}{v}_{d}$ causes the flow of particles. In this 
case, there is a transfer of electricity, heat (energy) etc. Consequently, in the presence of drift fields, the set of charge carriers in crystals becomes a large canonical nonequilibrium ensemble with a variable number of particles. Such a large canonical ensemble, as shown in [7], taking into account the spin degeneracy, is characterized by a large thermodynamic Gibbs potential:

$$
\left.\Omega=-2 k T \sum_{p} \ln \left\{1+\exp \left(\frac{\mu+\Delta \mu \underset{p}{r}-\varepsilon \underset{p}{r}}{k T}\right)\right\}=-2 V \int_{0}^{\infty} \frac{G(\varepsilon)}{\exp \left(\frac{\varepsilon-\mu-\Delta \mu r}{p}\right)+1}\right)^{r} d \varepsilon
$$

In equation (2) $\dot{p}$ - wave vector of the charge carrier, $\varepsilon_{p}^{r}$ - dispersion law of charge carriers, a $\Delta \mu \underset{p}{r}-$ the change in the chemical potential of one particle under the perturbations that move out a crystal from the state of thermodynamic equilibrium. When there are no such perturbations $\Delta \mu \underset{p}{r}=0$.

The value of $\Delta \mu \underset{p}{r}$ was calculated in paper [7], where it is shown that $\Delta \mu(\dot{p})$ is an odd function of $\dot{p}$ and has the following meaning for an isotropic crystal in a magnetic field with an induction vector $B$

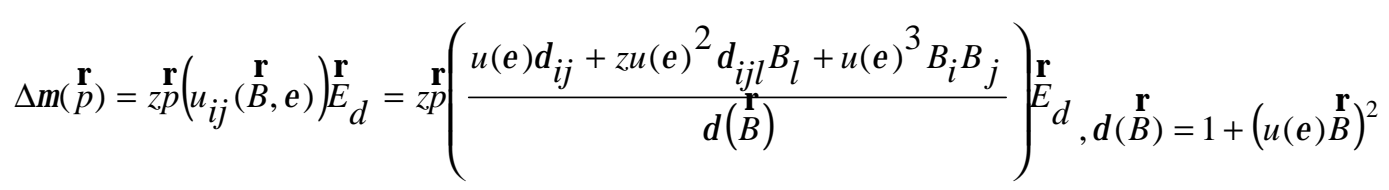

In formula (3), $\delta_{i j}$ and $\delta_{i j l}$ - Kronecker delta and Levi-Civita symbol, $u(\varepsilon)$ - dimensional scattering function that describes the influence of charge carrier scattering processes on crystal lattice defects on the kinetic properties of a crystal.

The cited papers show that in a crystal characterized by a large thermodynamic Gibbs potential (2), there are processes of transfer of electricity and heat, which are described by the following generalized equations of electrical conductivity and thermal conductivity:

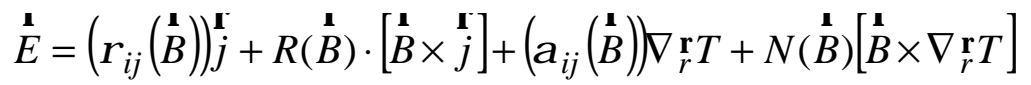

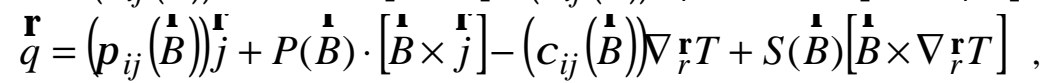

In equations (4) and (5), symmetric tensors $\left(\rho_{i j}\left(\begin{array}{l}1 \\ B\end{array}\right)\right)$, $\left(\alpha_{i j}(B)\right),\left(\pi_{i j}\left(\begin{array}{l}1 \\ B\end{array}\right)\right),\left(\chi_{i j}\left(\begin{array}{l}1 \\ B\end{array}\right)\right)$, respectively, are symmetric tensors of resistivity, Seebeck coefficient, Peltier coefficient, and thermal conductivity of the crystal, which must be experimentally determined or theoretically calculated. They are even functions of a magnetic induction vector.

The coefficients $R\left({ }^{\prime}\right)$ and $N\left({ }^{\prime}\right)$ are the coefficients of the transverse Hall and Nernst-Ettingshausen galvanomagnetic effects, and the coefficients $P(B)$ and $S(B)$ are the Nernst and Righi-Leduc transverse thermomagnetic effects. In isotropic crystals, these coefficients are scalar even functions of magnetic induction, i.e. $\quad R\left({ }^{\prime}\right)=R\left(-B^{\prime}\right), \quad P\left(B^{\prime}\right)=P\left(-B^{\prime}\right)$, $N(\stackrel{\dot{B}}{)}=N(-\stackrel{\dot{B}}{)}, S(\stackrel{\dot{B}}{B})=S(-\stackrel{\dot{B}}{)}$.

Analysis of equations (4), (5) shows that an isotropic crystal placed in a magnetic field becomes anisotropic, and the relatively simple phenomena of electrical conductivity and thermal conductivity in the crystal are complicated. In this case, additional transverse galvanic and thermomagnetic effects appear.

The galvanomagnetic effects are due to the action of the magnetic field on the ohmic part of the electric current, and the thermomagnetic effects on the thermal part, according to the generalized conductivity equation (4).

Kinetic tensors and coefficients in equations (4), (5), in addition to determining the nature of the important kinetic properties of the conducting medium, have wide application in the problems of predicting synthesized crystals with given properties.

The magnetic field $B$ in the crystal is considered weak if the condition is satisfied $(u(\varepsilon) B)^{2}<<1$. Under this condition, or in the absence of a magnetic field, all four kinetic tensors degenerate into scalars, and then all the important kinetic properties of an isotropic crystal are 
described by a set of such scalars: $\rho\left(\mu^{\bullet}, T\right), \alpha\left(\mu^{\bullet}, T\right)$, $\pi\left(\mu^{\bullet}, T\right), \chi\left(\mu^{\bullet}, T\right), R\left(\mu^{\bullet}, T\right), N\left(\mu^{\bullet}, T\right), P\left(\mu^{\bullet}, T\right)$, $S\left(\mu^{\bullet}, T\right)$

All these kinetic coefficients for crystals with macroscopic sizes, as shown in [7-9], can be calculated using the design functional:

$$
J\left(i, j, \mu^{\bullet}, T\right)=\int_{0}^{\infty} x^{i} u(x, T)^{j} G(x, T)\left(-\frac{d f_{0}}{d x}\right) d x
$$
$\varepsilon(\dot{p})$.

The functional (6) depends on the dispersion law

The dispersion law is an analytical formula $\varepsilon(\dot{p})$ that describes the dependence of the charge carrier energy of a crystal on its quasi-momentum $\dot{p}$.

The dispersion law $\varepsilon(\dot{p})$ is a complex quantum mechanical problem. For isotropic crystals, quantum mechanical studies substantiate the following general isotropic dispersion law:

$$
\frac{p_{x}^{2}+p_{y}^{2}+p_{x}^{2}}{2 m m_{n}}=\frac{p^{2}}{2 m m_{n}}=E(\varepsilon)
$$

In equation (7) $E(\varepsilon)$ - the homogeneous quantummechanical first-order energy function with respect to a parameter $k T, m$ - electron mass, $m_{n}-$ the relative effective mass of the crystal charge carrier.

The function $E(\varepsilon)$ is a homogeneous of first-order if for all values of $k T$-parameter the equation holds $E(k T \varepsilon)=k T E(\varepsilon)$.

The above mentioned analysis of statistical calculations of the thermal and kinetic properties of crystals showed that they all have an analytical dependence on the dispersion law (7), from which all the calculated parameters of the algorithmic calculation formulas have an analytical dependence. The following parameters include:

$g(\varepsilon)$ - the density of energy levels in the allowed energy zones of charge carriers in crystals,
$G(\varepsilon)=\int_{0}^{\varepsilon} g(\varepsilon) d \varepsilon$, scattering function $u(\varepsilon)$ that describes the influence of charge carrier scattering processes on crystal lattice defects on the kinetic properties of crystals. For the dispersion law (7) these values are the following:

$$
\begin{gathered}
G(\varepsilon)=\frac{8}{3 \sqrt{\pi}}\left(\frac{2 \pi m m_{n}}{h^{2}}\right)^{3 / 2} \mathrm{E}^{3 / 2}(\varepsilon) \\
g(\varepsilon)=\frac{d G(\varepsilon)}{d \varepsilon}=\frac{4 \pi}{\sqrt{\pi}}\left(\frac{2 \pi m m_{n}}{h^{2}}\right)^{3 / 2} \mathrm{E}^{1 / 2}(\varepsilon) \frac{d \mathrm{E}}{d \varepsilon} \\
G(x, T)=N_{C}(T) \cdot \mathrm{E}^{3 / 2}(x) \\
g(x, T)=\frac{3}{2} \frac{1}{k T} N_{C}(T) \mathrm{E}^{1 / 2}(x) \frac{d \mathrm{E}}{d x} \\
N_{C}(T)=\frac{8}{3 \sqrt{\pi}}\left(\frac{2 \pi m m_{n} k T}{h^{2}}\right)^{3 / 2} \\
u(\varepsilon)=u^{(r)}(T) p^{(2 r-3)}\left(\frac{d \varepsilon}{d p}\right)^{2}= \\
u^{(r)}(T) p^{(2 r-3)}\left(\frac{d p}{d \varepsilon}\right)^{-2} \\
u(x, T)=U(r, T) \cdot \frac{E^{(r-1 / 2)}(x)}{\left(\frac{d E(x)}{d x}\right)} \\
\left.u^{2}\right)
\end{gathered}
$$

In the latter formula $U(r, \mathrm{~T})$ - dimensional function of temperature with dimension of mobility, it is described by the following equation:

$$
U(r, T)=\left[\frac{1}{T}\left(U_{A} \delta(0, r)+U_{O} \delta(1, r)\right)+U_{I} \delta(2, r)\right]\left(m_{n}\right)^{(r-5 / 2)} T^{(r-1 / 2)}
$$

where $U_{A}, U_{O}, U_{I}$ - dimensional crystal constants that depend on the nature of the crystal and the nature of the charge carrier scattering mechanisms on crystal lattice defects, and $\delta(m, n)$ - Kronecker delta: $\delta(m, n)=1$, if $m=n$, $\delta(m, n)=0$, if $m \neq n ; r$ - scattering factor, $r=0$ - for scattering on acoustic phonons of the crystal lattice, $r=1-$ for scattering on optical phonons of the crystal lattice, $r=2$ - for scattering on ionized impurity atoms of the crystal lattice.

For the dispersion law (7), the design functional $J\left(i, j, \mu^{\bullet}, T\right)$ equals:

$$
\begin{aligned}
& J\left(i, j, \mu{ }^{\bullet}, T\right)=\int_{0}^{\infty}\left(\frac{\varepsilon}{k T}\right)^{i} u(\varepsilon)^{j} G(\varepsilon)\left(-\frac{d f_{0}}{d \varepsilon}\right) d \varepsilon=U(r, T)^{j} N_{C}(T) \int_{0}^{\infty} x^{i} \frac{E(x)^{(r j-j / 2+3 / 2)}}{\left(\frac{d E(x)}{d x}\right)^{2 j}}\left(\frac{d f_{0}}{d x}\right) d x= \\
& =U(r, T)^{j} N_{C}(T) I\left(i, j, \mu^{\bullet}, T\right),
\end{aligned}
$$


where dimensionless integral $I\left(i, j, \mu^{\bullet}, T\right)$ equals:

$$
I\left(i, j, \mu^{\bullet}, \beta(T)\right)=\int_{0}^{\infty} x^{i} \frac{E(x)^{(r j-j / 2+3 / 2)}}{\left(\frac{d E(x)}{d x}\right)^{2 j}}\left(\frac{d f_{0}}{d x}\right) d x
$$

For an isotropic parabolic dispersion law, the function $E(x)$ has next value:

$$
E(x)=x
$$

and for the Kane dispersion law, the function $E(x)$ is:

$$
\begin{aligned}
& E(x)=x\left(1+\frac{x}{E_{G}}\right)=x(1+\beta(T) x), \\
& \frac{d E(x)}{d x}=1+2 \beta(T) x
\end{aligned}
$$

where $E_{G}-$ crystal energy bandgap, $\beta(T)=\frac{k T}{E_{G}}-$ nonparabolicity parameter.

In narrow-bandgap crystals $E_{G}$, as a rule, the dispersion laws of electrons and holes are described by the nonparabolic Kane dispersion law. Therefore, the dimensionless integral $I\left(i, j, \mu^{\bullet}, T\right)$ for such crystals is:

$$
I\left(i, j, \mu \cdot \beta(T)=\int_{0}^{\infty} x^{i} \frac{\left.E(x)^{(r j-j / 2}+3 / 2\right)}{\left(\frac{d E(x)}{d x}\right)^{2 j}}\left(\frac{d f_{0}}{d x}\right) d x=\int_{0}^{\infty} x^{i} \frac{\left(x+\beta(T) x^{2}\right)^{(r j-j / 2+3 / 2)}}{(1+2 \beta(T) x)^{2 j}}\left(-\frac{\partial f_{0}}{\partial x}\right) d x\right.
$$

In wide-bandgap crystals $E_{G}$, the nonparabolicity parameter $\beta(T)=\frac{k T}{E_{G}}<<1 \rightarrow 0$, that is why this integral for such crystals has a Taylor approximation:

$$
\begin{gathered}
I\left(i, j, \mu^{\bullet}, \beta(T)\right)=\int_{0}^{\infty} x^{i} \frac{\left(x+\beta(T) x^{2}\right)^{(r j-j / 2+3 / 2)}}{(1+2 \beta(T) x)^{2 j}}\left(-\frac{\partial f_{0}}{\partial x}\right) d x \cong I\left(i, j, \mu^{\bullet}, 0\right)+ \\
+\left(\frac{d I\left(i, j, \mu^{\bullet}, \beta(T)\right)}{d \beta(T)}\right)_{\beta(T)=0} \times \beta(T)+\ldots=F_{l(i, j)}\left(\mu^{\bullet}\right)+(l(i . j)-4 j-i) F_{(l(i, j)+1)}\left(\mu^{\bullet}\right) \cdot \beta(T)+\ldots
\end{gathered}
$$

In this equation $F_{l(i, j)}\left(\mu^{\bullet}\right)$ - is a well-known Fermi integral with index $l(i, j)=i+r j-j / 2+3 / 2$, which has the following asymptotic properties: $F_{l}\left(\mu^{\bullet}\right) \cong \Gamma(l+1) \exp \left(\mu^{\bullet}\right)$, for $\mu^{\bullet}<-4$, where $\Gamma(l+1)$ - gamma Euler function; $F_{l}\left(\mu^{\bullet}\right) \cong\left(\mu^{\bullet}\right)^{l}$, for $\mu^{\bullet}>+4$.

These values are for the dimensionless integral $I\left(i, j, \mu^{\bullet}, \beta(T)\right)$ in case of isotropic crystals with parabolic dispersion law, where the nonparabolicity parameter $\beta(T)=\frac{k T}{E_{G}}=0$.

Equation (13) can be rewritten as:

$$
I\left(i, j, \mu^{\bullet}, \beta(T)\right) \cong F_{l(i, j)}\left(\mu^{\bullet}\right)\left[1+(l(i . j)-4 j-i) \frac{F_{((i, j)+1)}\left(\mu^{\bullet}\right)}{F_{l(i, j)}\left(\mu^{\bullet}\right)} \cdot \beta(T)\right]
$$

Such an approximation will be adequate if the condition is met:

$$
(l(i . j)-4 j-i) \frac{F_{((i, j)+1)}\left(\mu^{\bullet}\right)}{F_{l(i, j)}\left(\mu^{\bullet}\right)} \cdot \beta(T)=F\left(i, j, \mu^{\bullet}\right) \cdot \beta(T)<1
$$

Under this condition, equation (14) can be approximated by a function that is convenient for calculations:

$$
I\left(i, j, \mu^{\bullet}, \beta(T)\right) \cong F_{l(i, j)}\left(\mu^{\bullet}\right) \exp \left(F\left(i, j, \mu^{\bullet}\right) \cdot \beta(T)\right)
$$




\section{Analysis of Experimental Thermoelectric Characteristics of Si- Based Crystals}

In papers[7-9], the formulas of kinetic properties of crystals are analyzed in more detail for their application to the analysis of experimental data in order to elucidate the nature of the corresponding crystal properties. The general conclusion of such analysis has shown that the general algorithmic functional adequately describes the nature of the kinetic properties of crystals and it can be used to solve the problem of prediction of the crystal properties.

Using functional (6), the resistivity $\rho\left(\mu^{\bullet}, T\right)$ of the crystal, the thermoelectric Seebeck coefficient $\alpha\left(\mu^{\bullet}, T\right)$, and the concentration $n\left(\mu^{\bullet}, T\right)$ of crystal charge carriers with macroscopic dimensions and isotropic parabolic dispersion law are described by the following algorithmic formulas [9]:

$$
\begin{gathered}
\rho\left(\mu^{\bullet}, T\right)=\frac{1}{e n} \frac{J\left(0,0, \mu^{\bullet}, T\right)}{J\left(0,1, \mu^{\bullet}, T\right)}=\frac{1}{e} \cdot \frac{1}{J\left(0,1, \mu^{\bullet}, T\right)}=\frac{1}{e} \cdot \frac{1}{U(r, T) N_{C}(T) \int_{0}^{\infty} x^{(r+1)}\left(\frac{d f_{0}}{d x}\right) d x}= \\
=\frac{1}{e} \cdot \frac{1}{U(r, T) N_{C}(T) F_{(r+1)}\left(\mu^{\bullet}\right)} \\
\alpha\left(\mu^{\bullet}, T\right)=\left(\frac{k}{z e}\right)\left[\frac{J\left(1,1, \mu^{\bullet}, T\right)}{J\left(0,1, \mu^{\bullet}, T\right)}-\mu^{\bullet}\right]=\left(\frac{k}{z e}\right)\left[\frac{F_{(r+2)}\left(\mu^{\bullet}\right)}{F_{(r+1)}\left(\mu^{\bullet}\right)}-\mu^{\bullet}\right] \\
n\left(\mu^{\bullet}, T\right)=J\left(0,0, \mu^{\bullet}, T\right)
\end{gathered}
$$

We now apply formulas (16) and (17) for a brief analysis of the works [10-12]. In these works, we present experimental data of $\rho\left(\mu^{\bullet}, T\right)$ and $\alpha\left(\mu^{\bullet}, T\right)$ for $p$-SiGe whiskers with a microscopic thickness $d$, in which a spatial quantization of the energy spectrum of charge carriers is observed. In this regard, as shown in [9], such crystals have a correlation between kinetic properties and thickness $d$. This correlation arises because the total design functional (6) through the dispersion law has a dependence on $d$.

As shown in [9], the total design functional (6) under these conditions equals:

$$
\begin{gathered}
J\left(i, j, \mu^{\bullet}, d\right) \cong U(r, T)^{j} N_{C}(T) F_{l(i, j)}\left(\mu^{\bullet}\right) \exp \left(-F\left(i, j, \mu^{\bullet}, d\right) \cdot \frac{\lambda_{D}(T)}{d}\right), \\
F\left(i, j, \mu^{\bullet}, d\right)=\frac{3 \sqrt{\pi}}{4} \cdot \frac{F_{(i+r j-j / 2+1)}}{F_{(i+r j-j / 2+3 / 2)}} \cdot\left(1-\frac{1}{2 \sqrt{\pi}} \cdot \frac{\left.F_{(i+r j-j / 2}+1 / 2\right)}{\left.F_{(i+r j-} j / 2+1\right)} \cdot\left(\frac{\lambda_{D}(T)}{d}\right),\right.
\end{gathered}
$$

where

$$
\lambda_{D}(T)=\left(\frac{h^{2}}{2 m m_{p}}\right)^{1 / 2}=\frac{7.455 \cdot 10^{-6}}{\left(m_{p} \frac{T}{K}\right)^{1 / 2}}-\text { de Broglie }
$$

wavelength in $\mathrm{cm}$.

The condition under which functional (19) exists is described by the following equation:

$$
a>\frac{1}{2 \sqrt{\pi}} \cdot \frac{F_{(i+r j-j / 2+1 / 2)}\left(\mu^{\bullet}\right)}{F_{(i+r j-j / 2+1)}\left(\mu^{\bullet}\right)} \lambda_{D}
$$

The numerical coefficient in this equation for nondegenerate charge carriers with chemical potential $\mu^{\bullet} \leq-4$ is of the order of one. Under this condition, equation (20) takes the following form:

$$
\begin{aligned}
& d>\lambda_{D}(T), \quad \text { thus } \quad \frac{\lambda_{D}(T)}{d}<1, \\
& \left(\frac{\lambda_{D}(T)}{d}\right)^{2}<\frac{\lambda_{D}(T)}{d}<1
\end{aligned}
$$

and conforms to the general laws of quantum mechanics, because under the general observation conditions, the radius of a wire-like crystal cannot be equal to or less than the radius of a hydrogen atom.

Functional (19) reduces formulas (16) and (17) to the following form: 


$$
\begin{aligned}
\rho\left(\mu^{\bullet}, T, d\right) & =\frac{1}{e} \cdot \frac{1}{U(r, T) N_{C}(T) F_{(r+1)}\left(\mu^{\bullet}\right) \exp \left(-F\left(0,1, \mu^{\bullet}, d\right) \cdot \frac{\lambda_{D}(T)}{d}\right)} \\
\alpha\left(\mu^{\bullet}, T, d\right) & =\left(\frac{k}{z e}\left[\frac{F_{(r+2)}\left(\mu^{\bullet}\right) \exp \left(-F\left(1,1, \mu^{\bullet}, d\right) \cdot \frac{\lambda_{D}(T)}{d}\right)}{F_{(r+1)}\left(\mu^{\bullet}\right) \exp \left(-F\left(0,1, \mu^{\bullet}, d\right) \cdot \frac{\lambda_{D}(T)}{d}\right)}-\mu^{\bullet}\right]\right.
\end{aligned}
$$

Formulas (22) and (23) show that, in the presence of a spatial quantization of the energy spectrum of a charge carrier, the kinetic properties of such a crystal have an analytical dependence on its microscopic size $d$. This dependency is described by the following correlation function:

$$
K(d)=\exp \left(-F\left(i, j, \mu^{\bullet}, d\right) \cdot \frac{\lambda_{D}(T)}{d}\right)
$$

The general functional analysis of formulas (22)-(24) shows that they describe qualitatively and quantitatively the results of experimental studies of kinetic coefficients $\rho\left(\mu^{\bullet}, T, d\right)$ and $\alpha\left(\mu^{\bullet}, T, d\right)$ obtained in [10-12].

\section{Conclusions}

We have shown that the theoretical model of the kinetic properties of semiconductor crystals adequately describes the nature of the actual kinetic properties of crystals. The proposed general formulas of kinetic properties of crystals allowed qualitatively and quantitatively describing the experimental thermoelectric characteristics of Si-based whiskers.

Budzhak Ya.S. - Professor, Doctor of Science (PhD), Professor of the Department of Semiconductor Electronics;

Druzhinin A.A. - Professor, Doctor of Technical Sciences, Head of the Department of Semiconductor Electronics;

Nichkalo S.I. - Candidate of Technical Sciences, Senior Researcher, Department of Semiconductor Electronics.

[1] B.M. Askerov, Electron Transport Phenomena in Semiconductors (World Scientific, Singapore, 1994).

[2] H. Haug, A.-P. Jauho, Quantum Kinetics in Transport and Optics of Semiconductors (Springer, 1996).

[3] O. Madelung, Physics of III-V Compounds (Wiley, New York, 1964).

[4] K. Seeger, Semiconductor Physics (Springer-Verlag Wien, New York, 1973).

[5] P.Y. Yu, M. Cardona, Fundamentals of Semiconductors. Physics and Materials Properties (Springer, 2010).

[6] T. Pustelny, A. Opilski, B. Pustelny, Acta Physica Polonica A 114(6A), A183 (2008).

[7] Ya.S. Budzhak, T. Waclawski, Physics and Chemistry of Solid State 19(2), 134 (2018) (doi: 10.15330/pcss.19.2.134-138).

[8] Ya.S. Budzhak, Physics and Chemistry of Solid State 19(3), 217 (2018) (doi: 10.15330/pcss.19.3.217-221).

[9] Ya.S. Budzhak, A.O. Druzhinin, T.K. Waclawski, Physics and Chemistry of Solid State 20(2), 133 (2019) (doi: 10.15330/pcss.20.2.133-138).

[10] A. Druzhinin, I. Ostrovskii, Iu. Kogut, S. Nichkalo, T. Shkumbatyuk, Physica Status Solidi C 8(3), 867 (2011) (doi: 10.1002/pssc.200900266).

[11] A.P. Dolgolenko, A.A. Druzhinin, A.Ya. Karpenko, S.I. Nichkalo, I.P. Ostrovsky, P.G. Litovchenko, A.P. Litovchenko, Semiconductor Physics, Quantum Electronics \& Optoelectronics 14(4), 456 (2011).

[12] A. Druzhinin, I. Ostrovskii, N. Liakh-Kaguy et al., Proceedings of SPIE - The International Society for Optical Engineering 10445, Article number 1044556 (2017) (doi: 10.1117/12.2280895). 


\author{
Я.С. Буджак, А.О. Дружинін, С.І. Нічкало
}

\title{
Теоретичний аналіз експериментальних термоелектричних характеристик ниткоподібних кристалів на основі кремнію
}

Національний університет «Львівська політехніка», Львів, Україна, druzh@polynet.lviv.иа

У роботі показано, що коли в провідному кристалі створити електричне поле з напруженістю $\stackrel{1}{E}$ та градієнт температури $\nabla \underset{r}{\mathrm{r}} T$ і помістити цей кристал в магнітне поле з вектором індукції $B$, то в ньому виникають процеси перенесення носіїв заряду і теплоти, які описуються відомими узагальненими рівняннями електропровідності і теплопровідності. Тензори і скалярні коефіцієнти, які входять в склад цих рівнянь, це кінетичні властивості кристалів. Вони описують природу актуальних властивостей кристалів і мають широке прагматичне застосування в сучасній твердотілій електроніці, описується процес просторового квантування спектра та його плив на кінетичні властивості кристалів.

Ключові слова: потенціал Гіббса, хімічний потенціал, закон дисперсії, віскер, термоелектрика. 